\title{
Preparing electronic handbook for using active grammar during process of translation of technical texts into English
}

\author{
Valentin V. Mironov ${ }^{1, *}$, Alexandr I. Zavolokin ${ }^{1}$ and Aleksey K. Rozanov ${ }^{1}$ \\ ${ }^{1}$ Ryazan State Radio Engineering University, 390005, Ryazan, Russia
}

\begin{abstract}
This article considers some problems concerning process of teaching students a translation of technical texts from Russian into English. It also describes the structure of the electronic handbook for using active English grammar.
\end{abstract}

\section{Introduction}

For the last decades the English language has taken a particular position in scientific world. Nowadays specialists, postgraduates and young scientists must translate their reports, scientific articles and educational supplies into the English language. Many scientific conferences have international status with two working languages, for the present instance these are Russian and English. It stipulated the developing of electronic tutorials, which allow specialists, postgraduates and young scientists to master the technique of the translation from Russian into English. It is necessary to mark out the following moments:

- during translation of texts into another language postgraduates, young scientists and specialists have to deal with two languages, one of them being «hostile» for those, who is translating;

- almost all the students of technical specialties, postgraduates and young scientists are very seldom professional translators. It means that they don't know well how to use grammar rules and have pure vocabulary.

The first moment stipulates the necessity of practical mastery of grammar rules of the Russian and the English languages, stipulates the skills of finding grammatical equivalents during the process of translation. In this case the problem of scientific terms is urgent as their inadequate translation causes semantic errors. Someone abuses English words, for example he can use variety instead of manifold. The second moment requires to take account of the fact that postgraduates and young scientists have very little practice in translating texts from a natural language into a foreign one. They usually have a small vocabulary of foreign words, know the grammar badly. It makes them turn to dictionaries and the grammatical reference book and by doing this they spend much time.

\section{Analysis and discussion}

The circumstances above mentioned defined the aim and the structure of the electronic handbook for using active English grammar. So the aim of the given article is to acquaint the scientific society with such electronic application. The structure of it enables postgraduates and young scientists to translate their own articles and reports independently from somebody's help faster.

Thus electronic handbook for using active English grammar must:

1. Enable postgraduates and young scientists to find a necessary grammar rule fast at any moment of their translating a text;

2. Contain enough quantity patterns of translation, take into account technical directedness of students, postgraduates and young scientists;

3. Contain the list of frequently used formulas;

4. Have a simple and understandable interface.

To solve the first task the authors use the handbook on active English grammar [1]. Active grammar of the English language is used during the process of translation from Russian into English. Passive grammar is considered to be a grammar, which can be used during the process of translation from English into Russian. In our case active and passive grammar is true for those whose natural language is Russian. Active grammar necessarily implies a strict algorithm of actions during the process of translation.

All the English sentences can be built on one of 5 schemes of speaking/writing the main parts in it: the first one is for imperative sentences, the second and the fourth ones are for narrative sentences and for questions to a subject, the third and the fifth ones are for interrogative sentences [1, p. 23].

The algorithm given in the electronic handbook contains 5 steps [1, pp. 25-27]. Each one enables to solve particular grammar tasks of a concrete sentence:

1) This step means that it is necessary to define a type of a sentence, that is, whether a concrete sentence is

Corresponding author: mironov.v.v@rsreu.ru 
simple, complex, compound, exclamatory, imperative, narrative and interrogative.

2) The second step is necessary to define whether there is a modal verb in a sentence. In case of need the rule of modal verbs must be used. It is also necessary to define in what voice the verb must be used and whether the predicate is negative or affirmative.

3) Then one must define the mood (subjunctive or indicative), the tense and the aspect of the verb;

4) On coincidence of the first three steps with each other one must find the necessary scheme of a sentence;

5) The last step is that one must translate the sentence, it being necessary to define all the nouns with an article.

The works [1, 2] produce examples of defining schemes of constructing sentences, contain some nuances of applying concrete schemes and rules. A similar example is included into the developed electronic handbook.

The first section of the developed electronic handbook includes the following inlays:

- General information about English sentence;

- Brief survey of the schemes of English sentences according to the classification in $[1,2]$;

- Determinant of the necessary scheme;

- General view of the schemes of English sentences;

- Tense and aspect of a verb, auxiliary verbs and sequence of tenses;

- Algorithm of deducting a formula of a sentence;

- Detailed examination of an example of deducting a formula of an English sentence;

- Sequence of pronouncing main parts of an English sentence in imperative, narrative and interrogative ones; special cases;

-Main parts of sentence;

- Secondary parts of an English sentence;

- Verbs, their role and sequence of pronunciation of them in an English sentence;

- Modal verbs and their equivalents;

- Verbal ways of expressing tenses;

- Sequence of tenses in subordinate clauses;

- Aspects of actions in indicative mood;

- Subjunctive and imperative moods;

- Passive voice;

- non-finite verb forms;

- Infinitive;

- Gerund;

- Participle I and participle II;

- Impersonal and Nominal impersonal sentences;

- Turn of speech There be;

- Numerals;

- conjunctions and connective words;

- subordinate clauses;

- Rules of using articles;

The second section of the electronic handbook is an extensive set of sentences, phrases and standard turns of speech, which are typical for scientific and technical texts. They are ready to be used in English texts of articles and sentences.

All the patterns are categorized in the next way:

- Aim and tasks of research;

- Formulation of a problem;
- Object of a research;

- Decomposition of aims and tasks;

- Methods and approaches;

- Motives for analysis, levels of analysis;

- Characteristic of problems;

- Describing degrees of depth of research of problems;

- Review of literature;

- Expressing points of view;

- Expressions about theories;

- Advancement of hypothesis;

- Expressing generally accepted positions;

- Presentationofsources;

- Expressing and characterizing existing materials;

- Classification of material;

- References to sources;

- Beginning of process of informing;

- Continuation of process of informing;

- Expression of connection with subsequent exposition;

- Expression of connection with preceding exposition;

- Returning to what was told earlier;

- Pause in process of exposition;

- Corrections and explanations;

- Entry of additions;

- Activation of attention;

- Exposing own point of view;

- Illustration of theoretical positions;

- Reference to authorized sources;

- Expressing hypothesis;

- Explanations;

- Argumentations;

- Verification;

- Expressing assurance or doubt;

- Reconstruction of a complete picture;

- Exposing facts;

- Establishing of connection and difference between existing facts;

- Establishing of reasons and consequenceы;

- Abstraction of specific factors, features, phenomenon;

- Means of indication of historical time;

- Conclusion, consequences, summing up;

- Expression of gratitude.

The third section of the developed electronic resource contains reference material, which is useful while translating mathematical texts from Russian into English. The important feature of the material collected is that there is a transcription for each mathematical sign, abbreviation and Greek letters. Reference material of the electronic handbook is categorized in the following way:

- Greek alphabet and transcription;

- Table of mathematical signs and functions;

-Additional set of terms and notions connected with operations of addition, subtraction, multiplication, division, fractions, exponentiation;

- Fractions;

- relations and proportions;

- Mathematical equations;

- Examples of reading mathematical formulas;

- Table of abbreviation. 
The developed electronic handbook is a corrected and supplemented version of the textbook published earlier [2] and enables students, postgraduates and young scientists while working on translating Russian texts into the English language to use conveniences which are provided by the modern computers (contextual search, fast navigation about list of contents, possibility of duplication, availability of hyperlinks).

The electronic handbook is designed in the format Compiled HTML (CHM), distributed in the form of a file by usual copying out. Due to a wide prevalence of the format it is opened by any computer on the base of OC Windows and doesn't require a connection to Internet for work.

Figure 1 shows the surface appearance of contents directory of the electronic handbook.

The application of format CHM also allows to simplify the access to the sources which the author of the textbook [1] (Fig. 2).

Figure 3 illustrates the utility of interactive search in the electronic handbook.

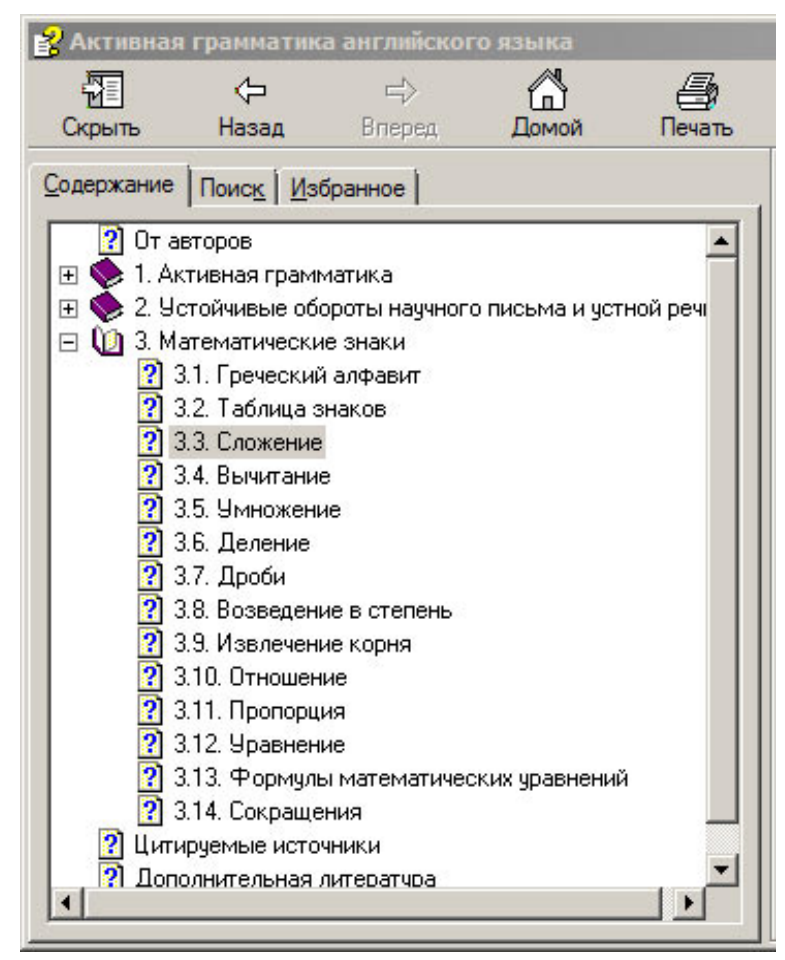

Fig. 1. The contents directory of the electronic handbook

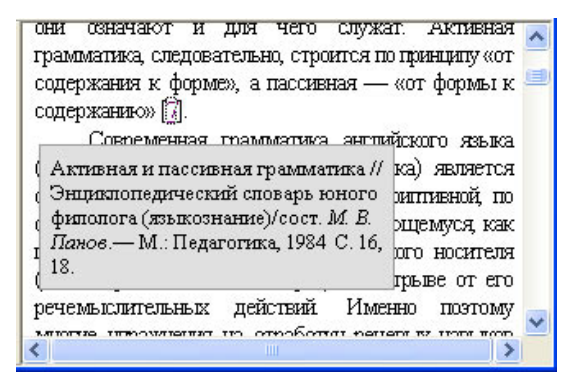

Fig. 2. Interactive references to sources in the electronic handbook.

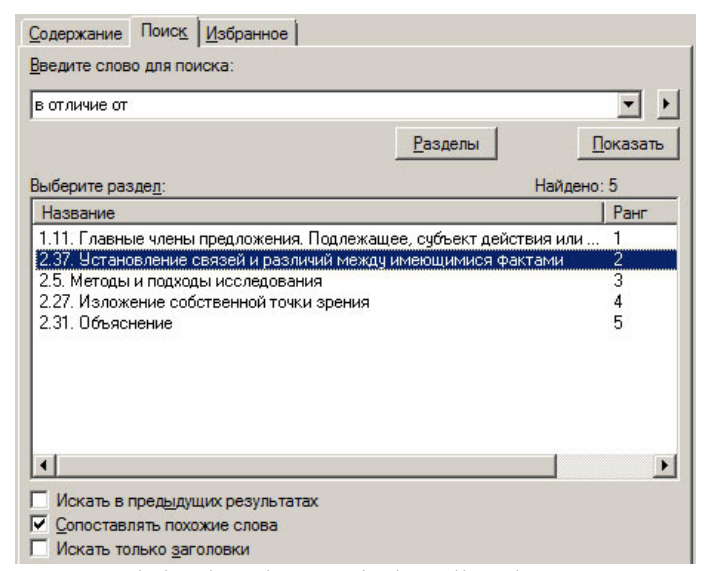

Fig. 3. Search in the electronic handbook.

\section{Conclusions}

Various approaches and personal skills are required for effective learning in modern society: for example, ability to make relations and see the sense between spheres of knowledge, concepts and ideas is one of the major skills ensuring effective activity in the modern world. Timely renewal of knowledge is a necessary feature of modern education. Moreover, this is a process of decisionmaking (ability to choose, analyze, organize, classify, evaluate incoming information), which presupposes high level of development of informative and cognitive independence of students' personality.

\section{References}

1. A.I. Zavolokin, V.V. Mironov. Active grammar of the English language (Moscow, 2015)

2. A.I. Zavolokin, V.V. Mironod, A.K. Rozanov. Informatization of Education and Science. 22, 149 (2014) 